\title{
Efficacy and safety of pyrotinib in advanced lung adenocarcinoma with HER2 mutations: a multicenter, single-arm, phase II trial
}

Zhengbo Song ${ }^{1 \dagger}$, Yuping $\mathrm{Li}^{2+}$, Shiqing Chen ${ }^{3+}$, Shenpeng Ying ${ }^{4 \dagger}$, Shuguang $\mathrm{Xu}^{5}$, Jianjin Huang ${ }^{6}$, Dan $\mathrm{Wu}^{7}$, Dongqing Lv $v^{8}$, Ting Bei ${ }^{3}$, Shuxun Liu' ${ }^{9}$, Xiaoping Huang ${ }^{10}$, Congying Xie ${ }^{11}$, Xiaoyu Wu ${ }^{12}$, Jianfei Fu ${ }^{13}$, Feng Hua ${ }^{14}$, Wenxian Wang ${ }^{15}$, Chunwei $\mathrm{Xu}^{16}$, Chan Gao ${ }^{3}$, Shangli Cai ${ }^{3}$, Shun Lu ${ }^{17}$ and Yiping Zhang ${ }^{16^{*}}$ (D

\begin{abstract}
Background: There is currently a lack of effective treatments for non-small cell lung cancer (NSCLC) patients harboring HER2 mutations. We examined the efficacy and safety of, and potential resistance mechanism to, pyrotinib, a pan-HER inhibitor, in advanced NSCLC carrying HER2 mutations.

Methods: In this multicenter, single-arm, phase II trial, stage IIIB-IV NSCLC patients harboring HER2 mutations, as determined using next-generation sequencing, were enrolled and treated with pyrotinib at a dose of $400 \mathrm{mg} /$ day. The primary endpoint was 6-month progression-free survival (PFS) rate, and secondary endpoints were objective response rate (ORR), PFS, overall survival (OS), disease control rate (DCR), and safety. The impact of different HER2 mutation types on sensitivity to pyrotinib and the potential of utilizing mutational profile derived from circulating tumor DNA (ctDNA) to predict disease progression were also explored.

Results: Seventy-eight patients were enrolled for efficacy and safety analysis. The 6-month PFS rate was 49.5\% (95\% confidence interval [Cl], 39.2-60.8). Pyrotinib produced an ORR of 19.2\% (95\% Cl, 11.2-30.0), with median PFS of 5.6 months $(95 \% \mathrm{Cl}, 2.8-8.4)$, and median OS of 10.5 months $(95 \% \mathrm{Cl}, 8.7-12.3)$. The median duration of response was 9.9 months $(95 \% \mathrm{Cl}, 6.2-13.6)$. All treatment-related adverse events (TRAEs) were grade 1-3 (all, 91.0\%; grade 3, 20.5\%), and the most common TRAE was diarrhea (all, 85.9\%; grade 3, 16.7\%). Patients with exon 20 and non-exon 20 HER2 mutations had ORRs of $17.7 \%$ and $25.0 \%$, respectively. Brain metastases at baseline and prior exposure to afatinib were not associated with ORR, PFS, or OS. LOSS of HER2 mutations and appearance of amplification in HER2 and EGFR were detected upon disease progression.
\end{abstract}

Conclusions: Pyrotinib exhibited promising efficacy and acceptable safety in NSCLC patients carrying exon 20 and non-exon 20 HER2 mutations and is worth further investigation.

Trial registration: Chinese Clinical Trial Registry Identifier: ChiCTR1800020262

Keywords: HER2 mutations, Non-small cell lung cancer, Pyrotinib, Efficacy, Resistance mechanism

\footnotetext{
* Correspondence: yi_ping_zhang@163.com

'Zhengbo Song, Yuping Li, Shiqing Chen and Shenpeng Ying contributed equally to this work.

${ }^{16}$ Department of Medical Oncology, Zhejiang Cancer Hospital, 1 East Banshan Road, Hangzhou 310022, China

Full list of author information is available at the end of the article
}

C The Author(s). 2022 Open Access This article is licensed under a Creative Commons Attribution 4.0 International License, which permits use, sharing, adaptation, distribution and reproduction in any medium or format, as long as you give appropriate credit to the original author(s) and the source, provide a link to the Creative Commons licence, and indicate if changes were made. The images or other third party material in this article are included in the article's Creative Commons licence, unless indicated otherwise in a credit line to the material. If material is not included in the article's Creative Commons licence and your intended use is not permitted by statutory regulation or exceeds the permitted use, you will need to obtain permission directly from the copyright holder. To view a copy of this licence, visit http://creativecommons.org/licenses/by/4.0/. The Creative Commons Public Domain Dedication waiver (http://creativecommons.org/publicdomain/zero/1.0/) applies to the data made available in this article, unless otherwise stated in a credit line to the data. 


\section{Background}

HER2-mutated non-small cell lung cancer (NSCLC) can only obtain limited clinical benefit from targeted therapies such as pan-HER tyrosine kinase inhibitors (TKIs) or TKIs targeting EGFR/HER1 or HER2 [1-3]. Although adotrastuzumab emtansine (T-DM1) and fam-trastuzumab deruxtecan-nxki (T-DXd) are recommended as treatment options for advanced HER2-mutant NSCLC patients by the National Comprehensive Cancer Network (NCCN) guidelines based on ORRs of $44 \%(N=18)$ and $72.7 \%(N$ $=11$ ), respectively in advanced HER2-mutant lung adenocarcinomas, these two drugs have not been approved yet for treating this subset of patients $[4,5]$. Chemotherapy remains the current standard-of-care for HER2-mutated NSCLC; however, it typically yields an ORR of 10-43.5\% (1st-line, 43.5\%; 2nd-line, 10\%) and a PFS of 4.3-6 months (1st-line, 6 months; 2nd-line, 4.3 months) [6, 7]. Therefore, there exists an unmet need for effective HER2targeting therapies to improve patients' outcomes. Multiple NSCLC trials are ongoing to evaluate other novel TKIs, including tarloxotinib (NCT03805841), TAK-788 (NCT02716116), and poziotinib (NCT03318939; NCT04 044170) [8].

Pyrotinib is an oral, irreversible pan-HER TKI, which has been adopted as the combination partner of capecitabine for treating advanced HER2- positive breast cancer in China [9]. In patient-derived lung cancer xenograft mouse models harboring HER2 exon 20 insertions, pyrotinib demonstrated stronger antitumor activities than TDM1 or afatinib [10]. In a phase II study $(N=60)$ conducted by Zhou $C$ et al., chemotherapy-treated NSCLC patients with HER2 mutations within exon 20 and 19 achieved an ORR of $30 \%$ upon pyrotinib, with mPFS of 6.9 months and median overall survival (mOS) of 14.4 months [11]. Evidence regarding efficacy and safety of pyrotinib remains to be confirmed in larger sample sizes, particularly in patients with HER2 mutations outside of exon 20. Moreover, the underlying mechanism of resistance to pyrotinib and its efficacy in patients who had brain metastases and prior exposure to anti-HER2 therapy has not been well elucidated.

The aim of this study was to evaluate the efficacy and safety of pyrotinib in advanced NSCLC patients harboring HER2 mutations. The impact of different HER2 mutation types on sensitivity to pyrotinib, the association between baseline characteristics and response, and the potential of utilizing mutational profile information derived from circulating tumor DNA (ctDNA) to predict disease progression were also explored.

\section{Methods}

\section{Patients}

Patients were recruited at 11 Chinese hospitals from December, 2018 until April, 2020. Patients were enrolled if they were 18 years or older and had histocytologically confirmed unresectable stage IIIB or IV NSCLC, HER2 mutations as determined using next-generation sequencing (NGS), an Eastern Cooperative Oncology Group (ECOG) performance status (PS) of 0-2, and at least one radiographically measurable lesion per Response Evaluation Criteria in Solid Tumors (RICIST) version 1.1 [12]. Exclusion criteria included having had undergone surgery, chemotherapy, or radiotherapy for NSCLC within 4 week before the study treatment. Written informed consent was provided by each patient before the onset of any trial-related treatment. The study protocol was approved by each site's institutional review board in accordance with the Declaration of Helsinki and Good Clinical Practice guidelines.

\section{Study design and treatment}

This is a multi-center, single-arm, phase II trial (Clinical trial registration: ChiCTR1800020262). Pyrotinib was administrated orally at $400 \mathrm{mg} /$ day within $0.5 \mathrm{~h}$ after breakfast until intolerable toxicity, disease progression, or discontinuation at the patient's request. In case of intolerable toxicity, the dose of pyrotinib was reduced to $320 \mathrm{mg}$ daily. Depending on sample availability, biopsy tissue sample or blood sample was obtained from each patient at baseline, followed by NGS analysis. Under patients' consents, blood samples were also collected from some patients upon disease progression for NGS analysis.

\section{Outcome assessment}

The primary end point was 6-month PFS rate, which was defined as the proportion of PFS at 6 months after the first dose of pyrotinib. Secondary endpoints included safety, ORR (the frequency of patients who have had obtained partial response [PR] or complete response [CR] at two consecutive evaluations at least 4 weeks apart), PFS (the time between the first dose of pyrotinib and disease progression or death due to any reason), OS (the time between the first dose of pyrotinib and death due to any reason), and disease control rate (DCR, the frequency of patients who have had achieved a stable disease or PR or CR for $\geq 6$ weeks before disease progression). Radiological assessment was conducted every six weeks in the first year, and every 9 weeks thereafter. Adverse events were assessed according to the National Cancer Institute Common Terminology Criteria for Adverse Events version 4.0. Upon disease progression, patients were followed up every 3 months until death. Exploratory endpoints included the association between different HER2 mutation types and ORR, PFS, OS, or DCR and the feasibility of using ctDNA to monitor disease progression. 


\section{Next-generation sequencing}

Baseline tissue or blood samples were subjected to NGSbased molecular profiling to identify gene aberrations including alterations in the driver genes (EGFR, $A L K$, ROS1, MET, BRAF, RET, HER2, and KRAS) recommended by NCCN guidelines for NSCLC, while blood samples obtained from patients at disease progression were analyzed using a panel spanning 150 cancer-related genes at 3D Medicines, Inc., a clinical laboratory accredited by the College of American Pathologists (CAP) and certified by the Clinical Laboratory Improvement Amendments (CLIA) laboratory (Additional file 1: Supplementary Method for NG S[13, 14], Additional file 2: Table S1).

\section{Statistical analysis}

According to previous study [15, 16], the 6-month progression-free rate of chemotherapy is hypothesized to be $30 \%$, then 67 patients would provide $80 \%$ power to detect a 6 -month progression-free rate of $45 \%$ at $5 \%$ alpha level. A total of 75 patients would need to be enrolled with the consideration of a dropout rate of $10 \%$.
All statistical analyses were performed using the SPSS statistical software (version 20.0) and GraphPad prism (version 7). PFS and OS were estimated using KaplanMeier curves, with $P$ value determined by a log-rank test. The difference in ORR and DCR between different groups were analyzed using the Fisher's exact test. Cox regression was applied for calculating hazard ratio (HR) and $95 \%$ confidence intervals (CIs). A two-tailed $P<0.05$ was defined as statistically significant.

\section{Results \\ Patients}

Between December, 2018 and April, 2020, 80 patients with HER2 mutations were screened for eligibility. Two patients were excluded for withdrawing informed consents before study treatment; hence, a total of 78 patients were enrolled in this study and were included in the efficacy and safety analyses (Fig. 1). As data cut-off (December 30, 2020), the median duration of follow-up time was 10.5 months (range, 1.0-21.4 months). A total of 19 patients were still on treatment and 59 patients discontinued treatment, among which 50 for disease

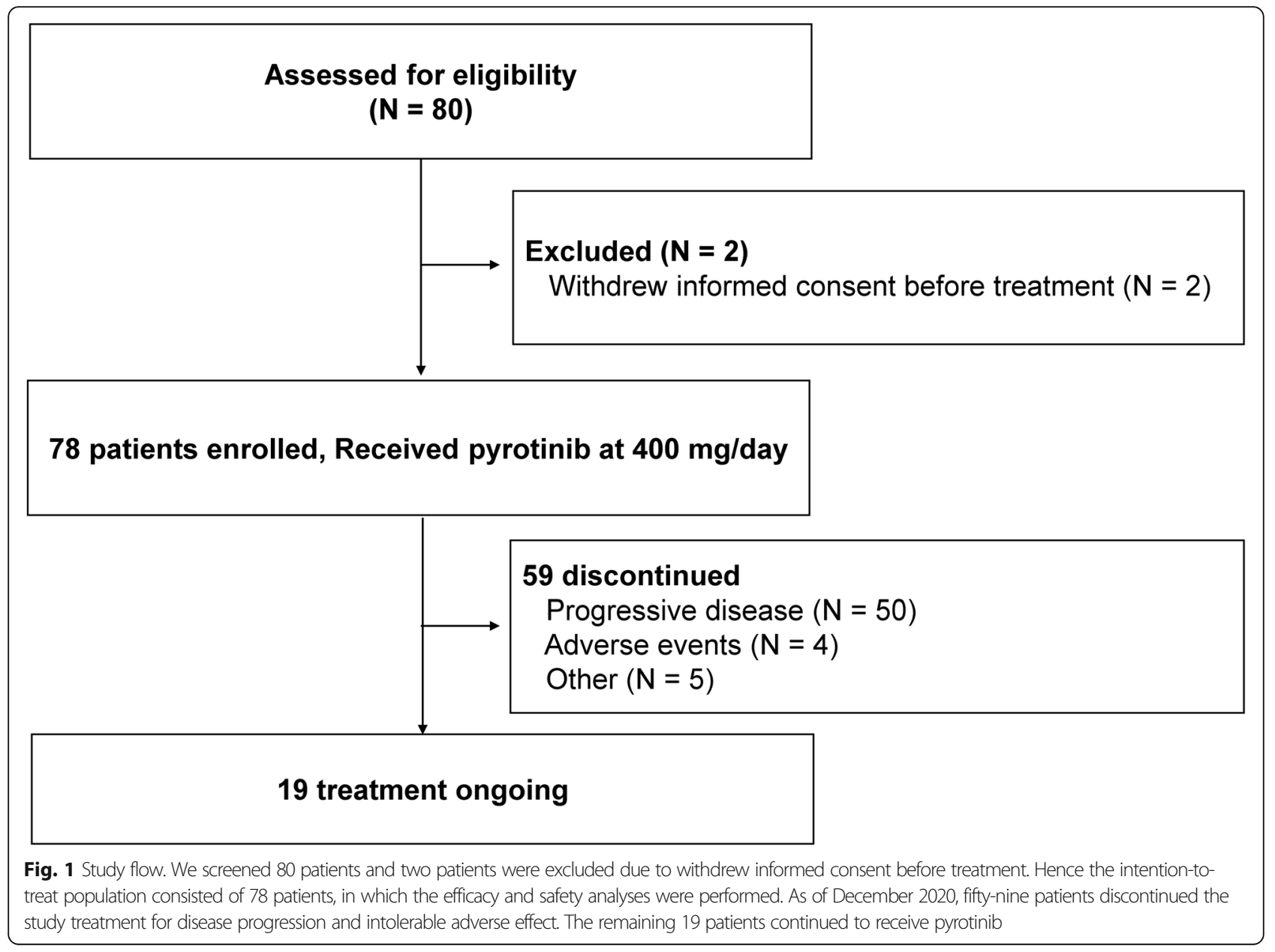


progression, 4 for intolerable adverse events, and the rest for other reasons.

Baseline characteristics were summarized in Table 1. The median age of the 78 patients was 62 years (range, 31-85 years). All patients had stage IV adenocarcinoma and $20(25.6 \%)$ had brain metastases. Seven patients (9.0\%) had an ECOG PS of 2 and the rest were 0-1. Most patients were non-smokers (65.4\%). Twenty-one

Table 1 Baseline characteristics

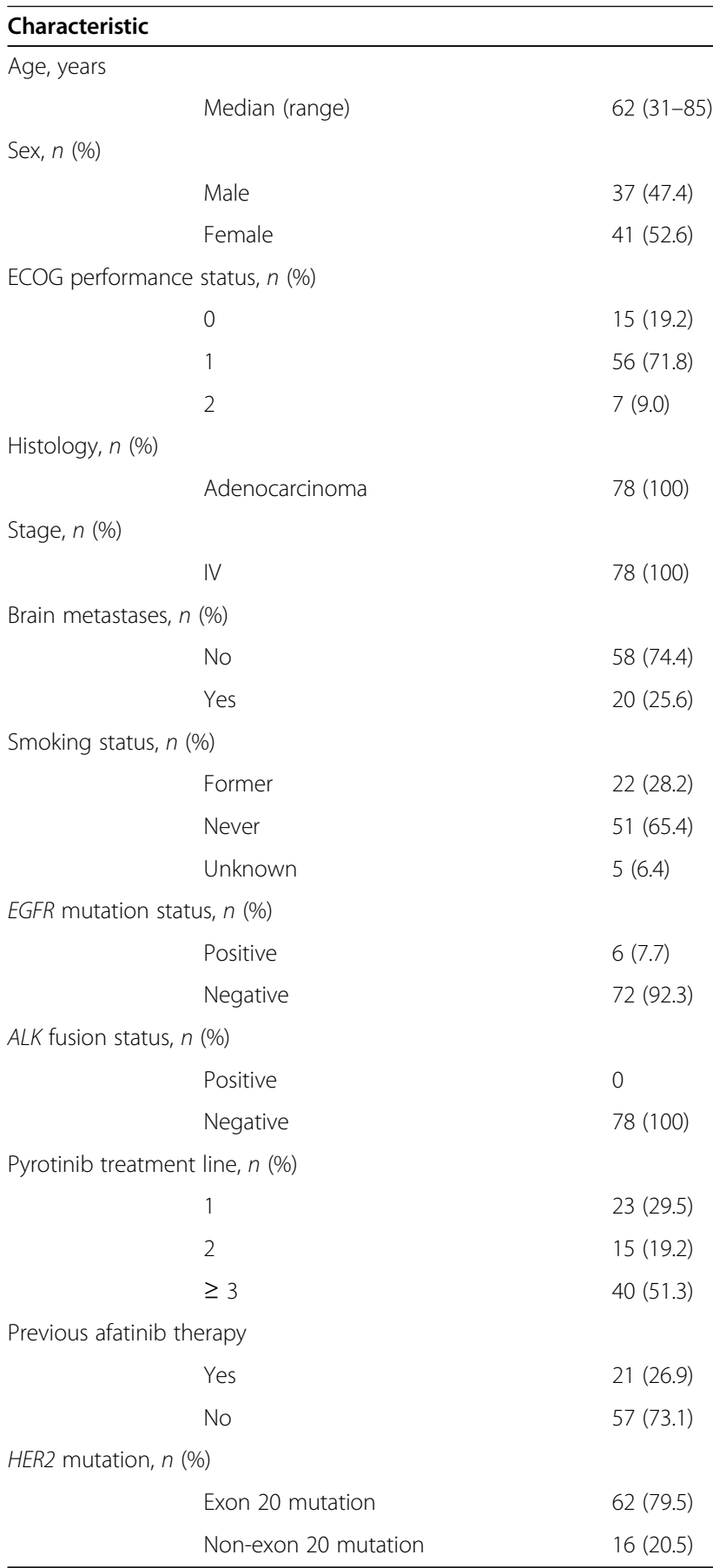

ECOG Eastern Cooperative Oncology Group patients had a prior exposure to afatinib (first-line, $N=$ 3 ; second-line or higher, $N=18$ ). The majority of the patients received pyrotinib in the second-line or higher (first-line, 29.5\%; second-line or higher, 70.5\%). Among the enrolled patients, 62 carried HER2 exon 20 mutations (79.5\%) while the other 16 patients (20.5\%) harbored mutations outside of exon 20. Of the 62 patients carrying exon 20 mutations, 42 and 11 patients had Y772_A775dup and G776delinsVC, respectively, and 9 carried other types of exon 20 mutations. Among the 78 patients, two patients harbored $\geq$ two HER2 mutations. A total of 81 HER2 mutations were detected at baseline, 73 fell in the kinase domain, three were in the transmembrane domain (TMD), three in extracellular domain, and the other two in other region of the coding region (Additional file 2: Fig. S1). HER2 mutation types identified at baseline were summarized in Additional file 2: Table S2.

\section{Efficacy}

As of December, 2020, the median duration of drug exposure was 5.6 months. A total of 50 PFS events and 40 deaths had occurred. The 6-month PFS rate was $49.5 \%$ (95\% CI, 39.2-60.8\%, Fig. 2). The 12-month PFS and OS rates were $28.4 \%$ and $38.6 \%$, respectively. The mPFS and mOS were 5.6 months (95\% CI, 2.8-8.4 months) and 10.5 months ( $95 \% \mathrm{CI}, 8.7-12.3$ months), respectively. Overall, 15 patients had a PR, for an ORR of 19.2\% (15/ 78; 95\% CI, 11.2-30.0\%), including 11 patients with HER2 mutations in exon 20, three in exon 19, and one in exon 17 (Table 2, Fig. 3). The median duration of response was 9.9 months (95\% CI, 6.2-13.6 months), and the disease control rate was $74.4 \%$ (58 of $78 ; 95 \% \mathrm{CI}$, $63.2-83.6 \%)$. Of these 15 patients who responded to pyrotinib, seven received pyrotinib as the first-line treatment, two were previously treated with afatinib, and three had brain metastases. All these 15 patients had a PS score of $0-1$.

When patients were stratified by baseline characteristics into comparison groups, we found that patients with a PS score of 2 displayed significantly worse OS than those with a PS score of $0-1$ (mOS, 10.7 vs. 6.1 months; HR, 0.28; 95\% CI, 0.11-0.75; $P=0.007$ ) (Additional file 2: Fig. S2). The ORRs of patients who received pyrotinib in the first-line and secondary-line or higher were $30.4 \%$ and $14.5 \%$, respectively (Additional file 2: Fig. S3). No significant difference in PFS or OS was observed among patients who received pyrotinib as the first-line treatment and those receiving pyrotinib in the secondary-line or higher setting (mPFS, 8.9 vs. 4.0 months; HR, 0.63; $95 \%$ CI, $0.33-1.18 ; P=0.144$; $\mathrm{OS}=12.5$ vs. 8.7 months; HR, 0.58; 95\% CI, 0.28-1.18; $P=0.125$ ) (Additional file 2: Fig. S4). The brain metastases at baseline and prior 
a

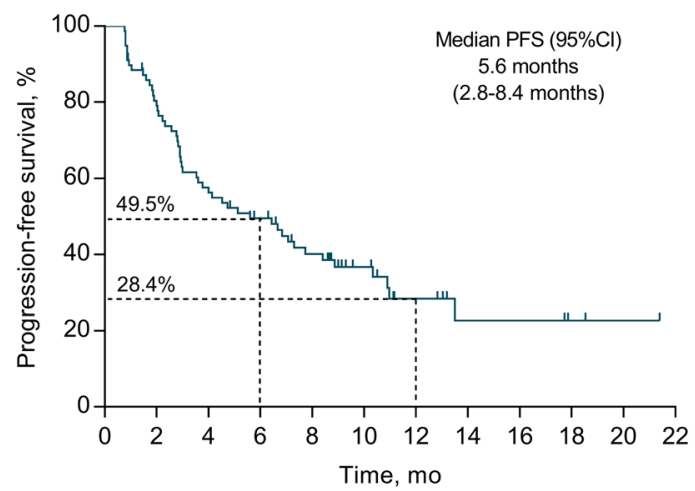

No. at risk: $\begin{array}{llllllllllll}78 & 60 & 43 & 34 & 25 & 16 & 8 & 4 & 4 & 2 & 1 & 0\end{array}$ b

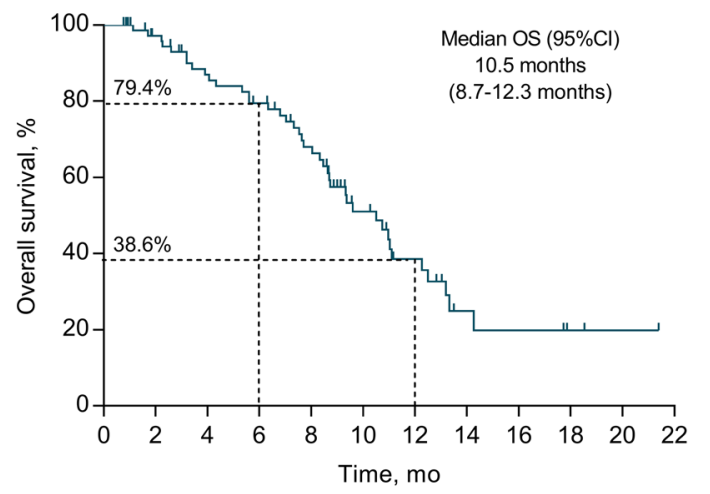

No. at risk: $\begin{array}{llllllllllll}78 & 68 & 58 & 51 & 40 & 23 & 13 & 5 & 4 & 2 & 1 & 0\end{array}$

Fig. 2 Kaplan-Meier survival curves of PFS and OS in pyrotinib treated NSCLC patients. PFS, progression-free survival; OS, overall survival; $95 \% \mathrm{Cl}$, 95\% confidence interval

exposure to afatinib were not significantly associated with ORR, PFS, or OS (Additional file 2: Fig. S2-Fig. S4).

Upon dissection by HER2 mutation types, the 62 patients harboring exon 20 mutations showed an ORR of 17.7\% (95\% CI, 9.2-29.5\%) (Additional file 2: Fig. S3, Table S3). The ORRs for the patients harboring Y772_ A775duplication, G776delinsVC, and other exon $20 \mathrm{mu}-$ tations were $23.8 \%$ (95\% CI, 12.1-39.5), $0.0 \%$ (95\% CI, 0-28.5), and $11.1 \%$ (95\% CI, 0.3-48.3), respectively. It was noteworthy that the ORR of the patients with nonexon 20 mutations reached $25.0 \%$, which was comparable as seen in the patients harboring exon 20 mutations ( $25.0 \%$ vs. $17.7 \% ; P=0.495$ ). Particularly, among the six patients with exon 19 mutations, three achieved PR, reaching an ORR to $50 \%$. Of these three PR patients

Table 2 Clinical response to pyrotinib in NSCLC patients with HER2 mutation

\begin{tabular}{ll}
\hline Variable & \\
\hline Best response, $n(\%)$ & $15(19.2)$ \\
Partial response & $43(55.1)$ \\
Stable disease & $20(25.6)$ \\
Progressive disease & $19.2(11.2-30.0)$ \\
Objective response rate, \% (95\% Cl) & $74.4(63.2-83.6)$ \\
Disease control rate, \% (95\% Cl) & $9.9(6.2-13.6)$ \\
Duration of response, median (95\% Cl) & \\
Progression-free survival & $50(64.1)$ \\
Events, $n$ (\%) & $5.6(2.8-8.4)$ \\
Median, months (95\% Cl) & \\
Overall survival & $40(51.3)$ \\
Events, $n$ (\%) & $10.5(8.7-12.3)$ \\
Median, months (95\% Cl)
\end{tabular}

Cl confidence interval carrying exon 19 mutations, two were treated with pyrotinib as first-line treatment. In addition, among the three patients with TMD mutations, the two patients carrying V658E substitution showed PFS of 2.9-5.6 months and OS of 5.3-5.6 months, while the patient harboring I655V had PFS and OS of 0.8 and 1.13 months, respectively (data not shown). No significant differences in PFS or OS were observed between patients who had exon 20 and non-exon 20 mutations (Additional file 2: Fig. S5).

Patients harboring co-mutations in driver genes such as EGFR, KRAS, BRAF, and ROS1 at baseline exhibited similar ORR $(30.0 \%$ vs. $17.6 \%, P=0.434)$ and mPFS (3.0 vs. 6.7 months; $P=0.294$ ) to and a poorer mOS (6.8 vs. 11.0 months; $P=0.017$ ) than their wildtype counterparts (Additional file 2: Fig. S3, Fig. S6). Patients with EGFR mutations had numerically inferior clinical outcomes than the EGFR-wild-type patients (ORR, 0 vs. $20.8 \%, P=0.590$; PFS, 3 vs. 6.4 months, $P=0.185)$. No difference was seen in ORR (19.4\% vs. $16.7 \%$; $P=1.000)$, PFS (5.4 vs. 14.0 months; $P=0.421)$, or OS (10.5 vs. NR months; $P=0.558)$ between patients without and with HER2 copy number amplification (CNA) at baseline (Additional file 2: Fig. S3, Fig. S6).

\section{Safety}

Treatment-related adverse events (TRAEs) of any grade occurred in 71 of 78 patients $(91.0 \%)$, most of which were grade 1 or 2 (Table 3). Diarrhea was the most common TRAE (85.9\%), followed by fatigue $(57.7 \%)$, anemia (35.9\%), dizziness (33.3\%), decreased appetite (32.1\%), hand-foot syndrome (32.1\%), and nausea (32.1\%). Sixteen patients suffered from grade 3 TRAEs (20.5\%), including 13 diarrhea (16.7\%), 2 anemia (2.6\%), and 1 fatigue $(1.3 \%)$. No grade 4 or higher TRAEs were 

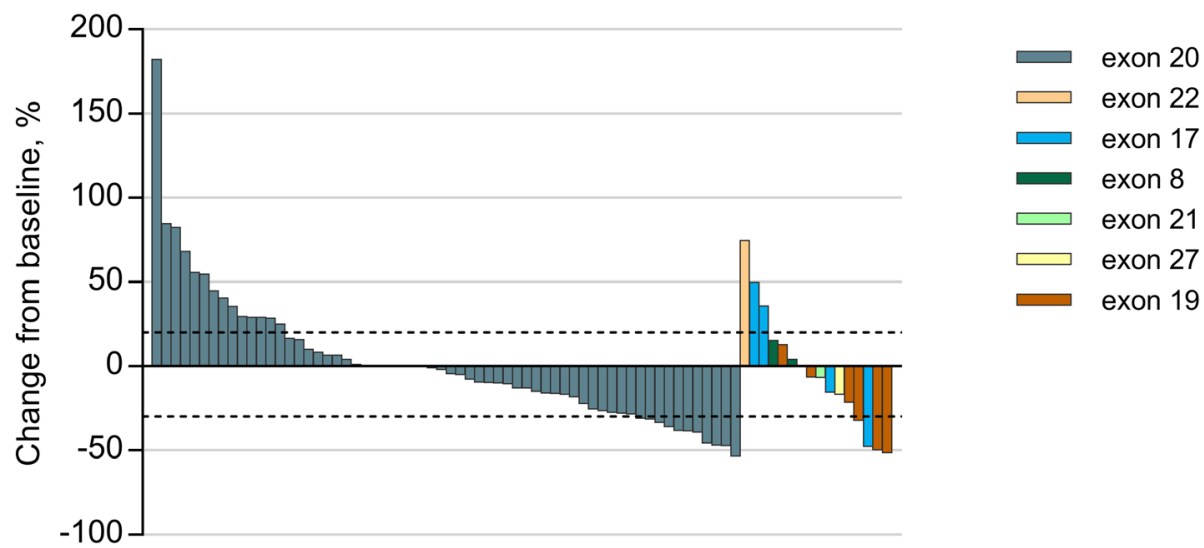

Fig. 3 Tumor regression from baseline in primary lesions. Different colors demote mutations in different exons

observed. Four patients discontinued treatment as a result of TRAEs, two for grade 3 diarrhea, one for grade 2 fatigue, and one for grade 2 decreased appetite, nausea, and vomiting. Two patients had a dose reduction due to intolerable toxicity.

Table 3 Treatment-related adverse events

\begin{tabular}{|c|c|c|c|c|}
\hline \multirow[t]{2}{*}{ Adverse event } & \multicolumn{4}{|c|}{ Pyrotinib $(n=78), n(\%)$} \\
\hline & All Grades & Grade 1 & Grade 2 & Grade 3 \\
\hline Any & $71(91.0)$ & $70(89.7)$ & $45(57.7)$ & $16(20.5)$ \\
\hline \multicolumn{5}{|c|}{ Occurring in $\geq 10 \%$ of patients } \\
\hline Diarrhea & $67(85.9)$ & $25(32.1)$ & $29(37.2)$ & $13(16.7)$ \\
\hline Fatigue & $45(57.7)$ & $39(50.0)$ & $5(6.4)$ & $1(1.3)$ \\
\hline Anemia & $28(35.9)$ & $18(23.1)$ & $8(10.3)$ & $2(2.6)$ \\
\hline Dizziness & $26(33.3)$ & $25(32.1)$ & $1(1.3)$ & \\
\hline Decreased appetite & $25(32.1)$ & $22(28.2)$ & $3(3.8)$ & \\
\hline Hand-foot syndrome & $25(32.1)$ & $22(28.2)$ & $3(3.8)$ & \\
\hline Nausea & $25(32.1)$ & $24(30.8)$ & $1(1.3)$ & \\
\hline WBC decreased & $19(24.4)$ & $13(16.7)$ & $6(7.7)$ & \\
\hline Blood creatinine increased & $19(24.4)$ & $19(24.4)$ & & \\
\hline Cough & $18(23.1)$ & $18(23.1)$ & & \\
\hline ALT increased & $17(21.8)$ & $17(21.8)$ & & \\
\hline Vomiting & $16(20.5)$ & $13(16.7)$ & $3(3.8)$ & \\
\hline Headache & $16(20.5)$ & $16(20.5)$ & & \\
\hline AST increased & $15(19.2)$ & $15(19.2)$ & & \\
\hline Hypokalemia & $14(17.9)$ & $14(17.9)$ & & \\
\hline Weight decreased & $12(15.4)$ & $11(14.1)$ & $1(1.3)$ & \\
\hline Pain & $12(15.4)$ & $12(15.4)$ & & \\
\hline Hyponatremia & $11(14.1)$ & $11(14.1)$ & & \\
\hline Chest distress & $10(12.8)$ & $9(11.5)$ & $1(1.3)$ & \\
\hline
\end{tabular}

$A L T$ alanine aminotransferase, $A S T$ aspartate aminotransferase, $W B C$ white blood cell

*No grade 4 or higher adverse events occurred
Feasibility of using ctDNA to monitor disease progression upon pyrotinib treatment

Of the 78 patients in the analysis cohort, twelve patients who acquired resistance to pyrotinib had blood samples available both at baseline and upon disease progression. These blood samples were subjected to NGS analysis to monitor disease progression. Concurrent HER2 CNA and EGFR CNA, which were not presented at baseline blood samples, were detected from two patients upon $\mathrm{PD}$, suggesting that co-occurrence of HER2 CNA and EGFR CNA may have played a role in resistance to pyrotinib. One of these two patients' representative CT images captured at baseline, best response, and PD are shown in Additional file 2: Fig. S7. Another four patients had a loss of HER2 mutation upon PD, rending it rational to speculate that the loss of HER2 mutations may confer resistance to pyrotinib. In addition, appearance of EGFR (p.E330K), KRAS (p.G12D), MET CNA, and BRAF CNA were also detected in three patients at PD (Additional file 2: Table S4). Since KRAS and BRAF are both downstream of HER2 in the RAS/RAF signaling pathway, our results suggested that gene alterations in the RAS/RAF pathway may serve as a potential mechanism of resistance to pyrotinib.

\section{Discussion}

HER2 mutations are rarely observed in NSCLC. There exists little evidence regarding effective treatment of NSCLC patients with $H E R 2$ mutations, especially those with non-exon 20 mutations. Herein, we reported the effect of pyrotinib in 78 advanced lung adenocarcinoma patients harboring different types of HER2 mutations. In the total population, pyrotinib produced 6-month PFS rate of $49.5 \%$, mPFS of 5.6 months, mOS of 10.5 months, and ORR of $19.2 \%$. In line with previous studies, the most common TRAE was diarrhea, and grade 3 diarrhea occurred in $16.7 \%$ of the patients. Among patients with 
HER2 mutations in different exons, patients harboring non-exon 20 aberrations achieved comparable ORR than those with exon 20 mutations. Patients who had brain metastases and prior exposure to anti-HER therapy could benefit from pyrotinib. Moreover, loss of HER2 mutations, appearance of HER2 amplification, and aberrations in EGFR, MET, KRAS, and BRAF were detected upon disease progression, suggesting their potential roles in the resistance to pyrotinib.

Chemotherapy, the current standard treatment for advanced NSCLC patients with HER2 mutations, typically elicits an ORR of $10 \%$ and an mPFS of 4.3 months in a second-line setting (6). TKIs targeting HER2 or pan-HER have been investigated for treating HER2-mutated lung cancer patients. However, afatinib, neratinib, and dacomitinib only elicited ORR of $7.7 \%, 3.8 \%$, and $12 \%$ [1-3]. The ORRs upon T-DM1 and T-DXd treatment could reach up to $44 \%(8 / 18)$ and $72.7 \%(8 / 11)$, respectively $[4,5]$. The mPFS of T-DM1-treated NSCLC patients as previously reported was 5.0 months, which was similar to that observed in the present study (5.0 vs. 5.6 months). Most recently, the results of the phase II study DESTINY-Lung trial were released in which T-DXd showed an ORR of 55\% (50/91) and mPFS of 8.2 months in patients with previously treated NSCLC with HER2 mutation [17]. Albeit encouraging anti-tumor activity, grade 4 and 5 TRAEs occurred upon T-DXd, whereas in our study, no grade 4 or 5 TRAEs were observed, suggesting that pyrotinib is safer than T-DXd $[5,17]$. Poziotinib, another promising antiHER2 TKI, has exhibited an ORR of $42 \%$ in HER2-mutated NSCLC patients $(N=12)$, causing grade 3 or 4 AEs in $66.7 \%$ of the patients [18].

Treatment of HER2-mutated NSCLC with pyrotinib has been previously reported. In phase II trials conducted by Wang Y et al. and Zhou $\mathrm{C}$ et al., treatment with pyrotinib was associated with ORRs of $53.3 \%$ and $30 \%$, and mPFSs of 6.4 months and 6.9 months in cohorts of 15 and 60 HER-mutated advanced NSCLC patients $[10,11]$. Both studies reported better efficacy than our observations (ORR, 19.2\%; PFS, 5.6 months). This could have been explained by the fact that our study enrolled patients with a PS score of $2(7 / 78,9 \%)$ whereas Zhou C's study only included patients with a PS score of $0-1$. A higher percentage of patients in our cohort had brain metastases at baseline (25.6\% vs. $20 \%)$ and more patients received pyrotinib in the third line or higher (51.3\% vs. $41.6 \%)$ than in their study. In addition, patients who had prior exposure to HER2-targeted drugs were also included in our study. Of note, the duration of response in the present study was 9.9 months, which was longer than 6.9 months documented in Zhou C's study.

The sensitivities to anti-HER2 TKIs in patients bearing different HER2 mutations were also distinct. In patients with HER2-mutated NSCLC, the major HER2 mutation type was exon 20 insertions, occurring in $1.5 \%$ of NSCL $\mathrm{C}$ and accounting for $90 \%$ of all NSCLC with HER2 mutations [19-22]. Previous studies have been mainly focusing on these insertions. Two prospective studies investigating pyrotinib employed the ADx HER2 Mutation Detection Kit for HER2 genotyping, which only allows for detection of exon 20 and 19 mutations [10, 11]. In our study, we utilized NGS to detect HER2 mutations, which was capable of identifying mutations outside of exons 20 and 19. Indeed, patients carrying mutations outside of exon 20 were also able to benefit from pyrotinib. A numerically higher ORR was observed among patients carrying non-exon 20 mutations, especially those carrying exon 19 mutations. These observations were consistent with previous findings that HER2 exon 20 insertions are less sensitive to currently available TKIs than mutations in other exons, potentially due to the structural difference of mutant in this exon from in others [19]. HER2 exon 20 insertions primarily affected two structural regions: the $\alpha \mathrm{C}$ - helix, comprising residues 770-774, and the loop region at residues 775-783 [20, 21, 23]. Structure-based comparison of behaviors between these variant types needs to be further studied.

Patients with HER2 exon 20 mutation Y772_A775dup, the most common HER2 mutation in NSCLC, failed to respond to afatinib and dacomitinib as reported [1, 24, 25]. Surprisingly, pyrotinib produced an ORR and a DCR of $23.8 \%$ and $78.6 \%$, respectively, in 42 patients harboring Y772_A775dup in our study [24, 25]. Consistent with the results of Zhou C's study, although none of the 11 patients carrying G776delinsVC achieved PR in our study, the DCR of this subset reached $63.6 \%$, which was similar to that of the other mutation types [11]. Clinical efficacy regarding anti-HER2 TKIs has been poorly investigated in patients with HER2 TMD mutations [26, 27]. In our study, three patients harbored HER2 TMD, including two with V659E and one with I655V. The PFS and OS of the patients with V659E was 2.9-5.6 months and 5.3-5.6 months, respectively. The other patient bearing $1655 \mathrm{~V}$, however, experienced $\mathrm{PD}$ three weeks after initiation of pyrotinib. Collectively, our results revealed variable efficacy of pyrotinib in NSCLC patients with different $H E R 2$ mutations and warrant further validation in larger randomized clinical trials.

Another point to be noted was the monitoring of acquired resistance to pyrotinib by using blood sample profiling, highlighting the importance of liquid biopsy in this setting. In this study, we also explored potential resistance mechanisms underlying disease progression upon pyrotinib. HER2 CNA was identified from two patients upon PD, consistent with a previous report that HER2 CNA conferred resistance to anti-HER2 TKIs in HER2-mutated NSCLC patients [28]. Of note, EGFR CNA was also detected from 
these two patients upon PD, indicating the concurrent HER2 CNA and EGFR CNA may engender resistance to pyrotinib. In another four PD patients, HER2 mutation, which existed at baseline, was not detected from the blood sample at $\mathrm{PD}$, rending it rational to speculate that the loss of HER2 mutations may engender resistance to pyrotinib as well. In addition, $M E T$ CNA, KRAS (p.G12D), BRAF CNA, and EGFR (p.E330K) were also detected from patients at PD. $M E T$ CNA has been reported to be associated with resistance to anti-HER2 TKIs in EGFR-mutant NSCL C, HER2-amplified breast cancer, and HER2-mutated NSCLC [28-30]. Based on these results, we propose that strategies combining pyrotinib and EGFR TKI or other TKIs targeting the above alternations might be a potential treatment option to vanquish resistance or potentiate the antitumor activities in treating this subset of patients.

Indeed, Rolfo $\mathrm{C}$ et al. summarized a series of novel agents that has potential against HER2-mutated NSCL $C$ [8]. Interestingly, the combinational treatment of a pan-HER inhibitor (neratinib) and T-DM1 or T-DXd induced a superior activity compared with T-DM1 alone [31]. Similarly, preclinical studies revealed that the novel pan-HER TKI poziotinib could up-regulate HER2 cell-surface expression and increase the activity of T-DM1 in tumors with HER2-mutation [32]. In addition, Bob $\mathrm{T}$. $\mathrm{Li}$ et al. reported that the combination of T-DM1 and irreversible pan-HER inhibitors (neratinib or afatinib) could enhance the duration of the responses in HER2-altered lung cancers [31]. Pyrotinib is an irreversible pan-HER inhibitor, also presenting promising activity in HER2-mutated NSCLC as observed in our study. Part of data of this trial (ChiCTR1800020262) was published recently which has shown the efficacy of pyrotinib in NSCLC patients with HER2 amplification (6-month PFS rate: 51.9\%, ORR: $22.2 \%$, mPFS: 6.3 months, mOS: 12.5 months) [33]. Therefore, a combination of T-DM1/TDXd and pyrotinib may become a potentially effective therapy for these HER2-altered patients. These results indicate that combining T-DM1/T-DXd and antiHER2 TKI might be a potential treatment option to increase antitumor activity or conquer resistance to targeted therapies. The above proposals are a ray of hope shining the future of patients with HER2 alternations.

Despite being the largest prospective study investigating pyrotinib effects in NSCLC, our study is still limited by the small sample size due to the low prevalence of $H E R 2$ mutations in NSCLC. Second, comparison with chemotherapy or other targeted therapies was not feasible due to a lack of control arm. The findings of the current study should be examined in larger randomized clinical trials.

\section{Conclusions}

Pyrotinib exhibited promising efficacy and acceptable safety in treating NSCLC patients with both exon 20 and non-exon 20 HER2 mutations.

\section{Abbreviations}

CAP: College of American Pathologists; Cl: Confidence interval; CLIA: Clinical laboratory improvement amendments; CNA: Copy number amplification; CR: Complete response; ctDNA: Circulating tumor DNA; DCR: Disease control rate; ECOG: Eastern Cooperative Oncology Group; HER2: Human epidermal growth factor receptor 2; HR: Hazard ratio; NCCN: National comprehensive cancer network; NGS: Next-generation sequencing; NSCLC: Non-small cell lung cancer; ORR: Objective response rate; OS: Overall survival; PFS: Progression-free survival; PR: Partial response; PS: Performance status; RICIST: Response evaluation criteria in solid tumors; T-DM1: Ado-trastuzumab emtansine; T-DXd: Fam-trastuzumab deruxtecan-nxki; TKI: Tyrosine kinase inhibitor; TMD: Transmembrane domain; TRAE: Treatment-related adverse event

\section{Supplementary Information}

The online version contains supplementary material available at https://doi. org/10.1186/s12916-022-02245-z.

\section{Additional file 1: Supplementary Methods.}

Additional file 2: Table S1-S4., Figure S1-S7. Table S1. List of genes in the 3DMed 150-gene panel. Table S2. HER2 mutations identified at baseline. Table S3. Clinical response to pyrotinib according to different HER2 mutation types. Table S4. The detected molecular alterations at baseline and progression. Fig. S1. HER2 mutational map at baseline. Green: receptor $L$ domain; red: furin-like cysteine rich region; blue: growth factor receptor domain IV; yellow: protein tyrosine kinase. Fig. S2. Survival curves of pyrotinib treated HER2-mutated NSCLC patients according to baseline characteristics. (A, B) progression-free survival (PFS) and overal survival (OS) according to the ECOG performance status. PS represents ECOG performance score. (C, D) PFS and OS of pyrotinib treated patients with or without brain metastasis. mPFS, median progression-free survival; mOS, median overall survival; HR, hazard ratio; 95\%Cl, 95\% confidence interval. Fig. S3 Objective response rate in pre-specific subgroups. Fig. S4 Survival curves of NSCLC patients treated with pyrotinib according to previous treatment. (A, B) progression-free survival (PFS) and overall survival (OS) according to the treatment lines of pyrotinib. (C, D) PFS and OS of patients according to the prior exposures to afatinib. MPFS, median progression-free survival; mOS, median overall survival; HR, hazard ratio; 95\% Cl, 95\% confidence interval. Fig. S5 Survival curves of pyrotinib treated NSCLC patients with different HER2 mutation. mPFS, median progression-free survival; mOS, median overall survival; HR, hazard ratio; 95\% Cl, 95\% confidence interval. Fig. S6 Survival curves of pyrotinib treated HER2-mutated NSCLC patients according to molecular characteristics. (A, B) progression-free survival and overall survival according to HER2 amplification. (C, D) PFS and OS of pyrotinib treated patients according to the occurrence of co-mutations in other driver genes. mPFS, median progression-free survival; mOS, median overall survival; HR, hazard ratio; 95\% Cl, 95\% confidence interval. Fig. S7 Pyrotinib resistance in a patient with HER2 and EGFR amplification. CT scans were performed at baseline ( 2 weeks before starting pyrotinib), best response ( 2 months after starting pyrotinib) and disease progression (7 months after starting pyrotinib), respectively. Mm, millimeter.

\section{Acknowledgements}

Pyrotinib was supplied by Jiangsu Hengrui Medicine Co, Ltd, Jiangsu, China. We would like to thank all the participating patients, their families and caregivers.

\section{Authors' contributions}

ZS, YL, SC1, and SY contributed equally to this study as co-first authors. YZ has full access to all of the data in the study and takes responsibility for the integrity of the data and the accuracy of the data analysis. Study concept and design: YZ, ZS, YL, SC1, and SY. Data acquisition, analysis: ZS, YL, SC1, SY, 
SX, JH, DW, DL, SL, XH, CX, XW, JF, FH, WW, CX, SC2, and SL. Data interpretation: ZS, YL, SC1, SY, and TB. Manuscript drafting: ZS, SC1, TB, and CG. Critical revision of the manuscript: $Y Z, Z S$, YL, SY, SC1, TB, and SC2. Statistical analysis: $\mathrm{ZS}$, and $\mathrm{SC} 1$. All authors read and approved the final manuscript.

\section{Funding}

This study was granted by Foundation of CSCO-Hengrui (Z. Song, Y-HR20190173). The funders had no role in study design, data collection and analysis, decision to publish, or preparation of the manuscript.

\section{Availability of data and materials}

The datasets used and/or analyzed during the current study are available from the corresponding author upon reasonable request.

\section{Declarations}

\section{Ethics approval and consent to participate}

The study protocol was approved by each site's institutional review board in accordance with the Declaration of Helsinki and Good Clinical Practice guidelines. Written informed consent was provided by each patient before the onset of any trial-related treatment.

\section{Consent for publication}

Not applicable.

\section{Competing interests}

Yiping Zhang has read the journal's policy, and the authors of this manuscript have the following competing interests: SC1 and TB are employees of 3D Medicines Inc. CG and SC2 contributed to this study when they were employees of 3D Medicines Inc. The remaining authors declare no conflict of interest.

\section{Author details}

'Department of Clinical Trial, Zhejiang Cancer Hospital, Hangzhou 310022, China. ${ }^{2}$ Department of Respiratory Diseases, The First Affiliated Hospital of Wenzhou Medical University, Wenzhou 325000, China. ${ }^{3}$ The Medical Department, 3D Medicines Inc., Shanghai 201114, China. ${ }^{4}$ Department of Radiotherapy, Taizhou Central Hospital, Affiliated Hospital of Taizhou University, Taizhou 318000, China. ${ }^{5}$ Department of Respiratory Disease, Ningbo Medical Center, Lihuili Eastern Hospital, Ningbo 315001, China. ${ }^{6}$ Department of Medical Oncology, the Second Affiliated Hospital, School of Medicine, Zhejiang University, Hangzhou 310009, China. ${ }^{7}$ Department of Thoracic Surgery, Cixi People Hospital, Ningbo 315300, China. ${ }^{8}$ Department of Respiratory Disease, Taizhou Hospital, Taizhou 317000, China. ${ }^{9}$ Department of Medical Oncology, Taizhou Cancer Hospital, Hangzhou 317500, China. ${ }^{10}$ Department of Respiratory Diseases, the Affiliated Ningbo No. 1 Hospital, School of Medicine, Ningbo University, Ningbo 315001, China. ${ }^{11}$ Department of Radiation and Medical Oncology, The Second Affiliated Hospital of Wenzhou Medical University, Wenzhou 325000, China. ${ }^{12}$ Department of Respiratory Diseases, Guangfu Hospital, Jinhua 321000, China. ${ }^{13}$ Department of Medical Oncology, Jinhua Central Hospital, Jinhua 321000, China. ${ }^{14}$ Department of Respiratory Diseases, Huzhou Central Hospital, Huzhou 313003, China. ${ }^{15}$ Department of Chemotherapy, Zhejiang Cancer Hospital, Hangzhou 310022, China. ${ }^{16}$ Department of Medical Oncology, Zhejiang Cancer Hospital, 1 East Banshan Road, Hangzhou 310022, China. ${ }^{17}$ Shanghai Lung Cancer Center, Shanghai Chest Hospital, Shanghai Jiao Tong University, Shanghai 20030, China.

Received: 12 October 2021 Accepted: 10 January 2022

\section{Published online: 01 February 2022}

\section{References}

1. Kris MG, Camidge DR, Giaccone G, Hida T, Li BT, O'Connell J, et al. Targeting HER2 aberrations as actionable drivers in lung cancers: phase II trial of the pan-HER tyrosine kinase inhibitor dacomitinib in patients with HER2-mutant or amplified tumors. Ann Oncol. 2015;26(7):1421-7. https://doi.org/10.1093/a nnonc/mdv186.

2. Dziadziuszko R, Smit EF, Dafni U, Wolf J, Wasag B, Biernat W, et al. Afatinib in NSCLC with HER2 mutations: results of the prospective, open-label phase II NICHE trial of European Thoracic Oncology Platform (ETOP). J Thorac Oncol. 2019;14(6):1086-94. https://doi.org/10.1016/j.jtho.2019.02.017.
3. Awada A, Colomer R, Inoue K, Bondarenko I, Badwe RA, Demetriou G, et al. Neratinib plus paclitaxel vs trastuzumab plus paclitaxel in previously untreated metastatic ERBB2-positive breast cancer: the NEfERT-T randomized clinical trial. JAMA Oncol. 2016;2(12):1557-64. https://doi.org/1 0.1001/jamaoncol.2016.0237

4. Li BT, Shen R, Buonocore D, Olah ZT, Ni A, Ginsberg MS, et al. Adotrastuzumab emtansine for patients with HER2-mutant lung cancers: results from a phase II basket trial. J Clin Oncol. 2018;36(24):2532-7. https://doi. org/10.1200/JCO.2018.77.9777.

5. Tsurutani J, Iwata H, Krop I, Jänne PA, Doi T, Takahashi S, et al. Targeting HER2 with trastuzumab deruxtecan: a dose-expansion, phase I study in multiple advanced solid tumors. Cancer Discov. 2020;10(5):688-701. https:// doi.org/10.1158/2159-8290.CD-19-1014.

6. Mazieres J, Barlesi F, Filleron T, et al. Lung cancer patients with HER2 mutations treated with chemotherapy and HER2-targeted drugs: results from the European EUHER2 cohort. Ann Oncol. 2016;27(2):281-6. https://doi. org/10.1093/annonc/mdv573.

7. Hartmann JT, Lipp HP. Toxicity of platinum compounds. Expert Opin Pharmacother. 2003:4(6):889-901. https://doi.org/10.1517/14656566.4.6.889.

8. Rolfo C, Russo A. HER2 mutations in non-small cell lung cancer: a Herculean effort to hit the target. Cancer Discov. 2020;10(5):643-5. https://doi.org/10.11 58/2159-8290.CD-20-0225.

9. Blair HA. Pyrotinib: First Global Approval. Drugs. 2018;78(16):1751-5. https:// doi.org/10.1007/s40265-018-0997-0.

10. Wang Y, Jiang T, Qin Z, Jiang J, Wang Q, Yang S, et al. HER2 exon 20 insertions in non-small-cell lung cancer are sensitive to the irreversible panHER receptor tyrosine kinase inhibitor pyrotinib. Ann Oncol. 2019;30(3):44755. https://doi.org/10.1093/annonc/mdy542.

11. Zhou C, Li X, Wang Q, Gao G, Zhang Y, Chen J, et al. Pyrotinib in HER2mutant advanced lung adenocarcinoma after platinum-based chemotherapy: a multicenter, open-label, single-arm, phase II study. J Clin Oncol. 2020;38(24):2753-61. https://doi.org/10.1200/JCO.20.00297.

12. Eisenhauer EA, Therasse P, Bogaerts J, Schwartz LH, Sargent D, Ford R, et al. New response evaluation criteria in solid tumours: revised RECIST guideline (version 1.1). Eur J Cancer. 2009;45(2):228-47. https://doi.org/10.1016/j.ejca.2 008.10.026.

13. Su D, Zhang D, Chen K, Lu J, Wu J, Cao X, et al. High performance of targeted next generation sequencing on variance detection in clinical tumor specimens in comparison with current conventional methods. J Exp Clin Cancer Res. 2017;36(1):121. https://doi.org/10.1186/s13046-0170591-4

14. Wang Z, Duan J, Cai S, Han M, Dong H, Zhao J, et al. Assessment of blood tumor mutational burden as a potential biomarker for immunotherapy in patients with non-small cell lung cancer with use of a next-generation sequencing cancer gene panel. JAMA Oncol. 2019:5(5):696-702. https://doi. org/10.1001/jamaoncol.2018.7098.

15. Fossella FV, DeVore R, Kerr RN, Crawford J, Natale RR, Dunphy F, et al. Randomized phase III trial of docetaxel versus vinorelbine or ifosfamide in patients with advanced non-small-cell lung cancer previously treated with platinum-containing chemotherapy regimens. The TAX 320 Non-Small Cell Lung Cancer Study Group. J Clin Oncol. 2000;18(12):2354-62. https://doi. org/10.1200/JCO.2000.18.12.2354.

16. Garon EB, Ciuleanu TE, Arrieta O, Prabhash K, Syrigos KN, Goksel T, et al. Ramucirumab plus docetaxel versus placebo plus docetaxel for second-line treatment of stage IV non-small-cell lung cancer after disease progression on platinum-based therapy (REVEL): a multicentre, double-blind, randomised phase 3 trial. Lancet. 2014;384(9944):665-73. https://doi.org/10.1 016/S0140-6736(14)60845-X.

17. Li BT, Smit EF, Goto $Y$, Nakagawa K, Udagawa H, Mazières J, et al. Trastuzumab deruxtecan in HER2-mutant non-small-cell lung cancer. N Engl J Med. 2021. https://doi.org/10.1056/NEJMoa2112431

18. Robichaux JP, Elamin YY, Vijayan RSK, Nilsson MB, Hu L, He J, et al. Pancancer landscape and analysis of ERBB2 mutations identifies poziotinib as a clinically active inhibitor and enhancer of T-DM1 activity. Cancer Cell. 2020; 37(3):420. https://doi.org/10.1016/j.ccell.2020.03.003.

19. Friedlaender A, Subbiah $\vee$, Russo A, Banna GL, Malapelle U, Rolfo C, et al. EGFR and HER2 exon 20 insertions in solid tumours: from biology to treatment. Nat Rev Clin Oncol. 2021;19(1):51-69. https://doi.org/10.1038/s41 571-021-00558-1.

20. Arcila ME, Chaft JE, Nafa K, Roy-Chowdhuri S, Lau C, Zaidinski M, et al. Prevalence, clinicopathologic associations, and molecular spectrum of ERBB2 
(HER2) tyrosine kinase mutations in lung adenocarcinomas. Clin Cancer Res. 2012;18(18):4910-8. https://doi.org/10.1158/1078-0432.CCR-12-0912.

21. Mazieres J, Peters S, Lepage B, et al. Lung cancer that harbors an HER2 mutation: epidemiologic characteristics and therapeutic perspectives. J Clin Oncol. 2013;31(16):1997-2003. https://doi.org/10.1200/JCO.2012.45.6095.

22. My Cancer Genome. ERBB2 exon 20 insertion. 2017. https://www.myca ncergenome.org/content/alteration/egfr-exon-20-insertion. Accessed 20 June 2021.

23. Wang SE, Narasanna A, Perez-Torres M, Xiang B, Wu FY, Yang S, et al. HER2 kinase domain mutation results in constitutive phosphorylation and activation of HER2 and EGFR and resistance to EGFR tyrosine kinase inhibitors. Cancer Cell. 2006;10(1):25-38. https://doi.org/10.1016/j.ccr.2006.05. 023.

24. Fang $W$, Zhao $S$, Liang $Y$, Yang $Y$, Yang $L$, Dong $X$, et al. Mutation variants and co-mutations as genomic modifiers of response to afatinib in HER2mutant lung adenocarcinoma. Oncologist. 2019;25(3):e545-54. https://doi. org/10.1634/theoncologist.2019-0547.

25. Zhao S, Fang W, Pan H, Yang Y, Liang Y, Yang L, et al. Conformational landscapes of HER2 exon 20 insertions explain their sensitivity to kinase inhibitors in lung adenocarcinoma. J Thorac Oncol. 2020;15(6):962-72. https://doi.org/10.1016/j.jtho.2020.01.020.

26. Bargmann $\mathrm{Cl}$, Hung MC, Weinberg RA. Multiple independent activations of the neu oncogene by a point mutation altering the transmembrane domain of p185. Cell. 1986;45(5):649-57. https://doi.org/10.1016/0092-8674 (86)90779-8.

27. Ou SI, Schrock AB, Bocharov EV, et al. HER2 transmembrane domain (TMD) mutations (V659/G660) that stabilize homo- and heterodimerization are rare oncogenic drivers in lung adenocarcinoma that respond to afatinib. J

Thorac Oncol. 2017;12(3):446-57. https://doi.org/10.1016/j.jtho.2016.11.2224.

28. Chuang JC, Stehr H, Liang Y, Das M, Huang J, Diehn M, et al. ERBB2mutated metastatic non-small cell lung cancer: response and resistance to targeted therapies. J Thorac Oncol. 2017;12(5):833-42. https://doi.org/10.101 6/j.jtho.2017.01.023.

29. Engelman JA, Zejnullahu K, Mitsudomi T, Song Y, Hyland C, Park JO, et al. MET amplification leads to gefitinib resistance in lung cancer by activating ERBB3 signaling. Science. 2007;316(5827):1039-43. https://doi.org/10.1126/ science.1141478.

30. Shattuck DL, Miller JK, Carraway KL 3rd, et al. Met receptor contributes to trastuzumab resistance of Her2-overexpressing breast cancer cells. Cancer Res. 2008;68(5):1471-7. https://doi.org/10.1158/0008-5472.CAN-07-5962.

31. Li BT, Michelini F, Misale S, Cocco E, Baldino L, Cai Y, et al. HER2-mediated internalization of cytotoxic agents in ERBB2 amplified or mutant lung cancers. Cancer Discov. 2020;10(5):674-87. https://doi.org/10.1158/2159-82 90.CD-20-0215.

32. Robichaux JP, Elamin YY, Vijayan RSK, Nilsson MB, Hu L, He J, et al. Pancancer landscape and analysis of ERBB2 mutations identifies poziotinib as a clinically active inhibitor and enhancer of T-DM1 activity. Cancer Cell. 2019; 36(4):444-57 e7. https://doi.org/10.1016/j.ccell.2019.09.001.

33. Song Z, Lv D, Chen SQ, Huang J, Li Y, Ying S, et al. Pyrotinib in patients with HER2-amplified advanced non-small cell lung cancer: a prospective, multicenter, single-arm trial. Clin Cancer Res. 2021. https://doi.org/10.1158/1 078-0432.CCR-21-2936.

\section{Publisher's Note}

Springer Nature remains neutral with regard to jurisdictional claims in published maps and institutional affiliations.

Ready to submit your research? Choose BMC and benefit from:

- fast, convenient online submission

- thorough peer review by experienced researchers in your field

- rapid publication on acceptance

- support for research data, including large and complex data types

- gold Open Access which fosters wider collaboration and increased citations

- maximum visibility for your research: over $100 \mathrm{M}$ website views per year

At BMC, research is always in progress.

Learn more biomedcentral.com/submissions 